DOI: $10.15393 / \mathrm{j} 3$.art.2019.7211

UDC $517.28,517.54,517.41$

V. V. STARKOV

\title{
THE JACOBIAN CONJECTURE: STRUCTURE OF KELLER MAPPINGS
}

\begin{abstract}
The Jacobian conjecture was first formulated by O. N. Keller in 1939. In the modern form it supposes injectivity of the polynomial mapping $f: \mathbb{R}^{n} \rightarrow \mathbb{R}^{n}\left(\mathbb{C}^{n} \rightarrow \mathbb{C}^{n}\right)$ under the assumption that $J_{f} \equiv$ const $\neq 0$. In this paper, we consider the structure of polynomial mappings $f$ with $J_{f} \equiv$ const $\neq 0$.
\end{abstract}

Key words: Jacobian conjecture, Keller mapping 2010 Mathematical Subject Classification: $14 R 15$

1. Introduction. Let $\mathcal{P}_{m}$ denote the set of all polynomials in $\mathbb{R}^{n}$ (or $\left.\mathbb{C}^{n}\right)$ of degree at most $m$. Let $P_{m}$ be the set of all polynomial mappings $F=\left(F_{1}, \ldots, F_{n}\right): \mathbb{R}^{n} \rightarrow \mathbb{R}^{n}$ (or $\left.\mathbb{C}^{n} \rightarrow \mathbb{C}^{n}\right), F_{k} \in \mathcal{P}_{m}(k=1, \ldots, n)$ degree $\operatorname{deg} F_{k} \leqslant m$. Denote the Jacobi matrix and the Jacobian of a mapping $F$ by $D F$ and $J_{F}$, respectively (in the complex case, $D F$ and $J F$ are complex). The Jacobian conjecture, formulated by Keller [3] in 1939, in the modern terms reads:

if $F \in P_{m}$ and $J_{F} \equiv$ const $\neq 0$, then $F$ is injective in $\mathbb{R}^{n}\left(\mathbb{C}^{n}\right)$.

Proof of the conjecture would allow to apply it widely in a number of branches of mathematics (e.g., [1], [4]). The conjecture is included in the list "Mathematical Problems for the Next Century" [7].

A significant amount of scientific publications have been devoted to this conjecture: see, e.g., [2]. In particular, it is proved for $F \in P_{2}$ for any $n$ in [10], checked for $n=2$ and $F \in P_{100}$ in [5]. However, up to date JC has been neither proved nor rejected for any $n$.

It is important to describe subsets of such mappings from $P_{m}$, that $J_{F} \equiv 1, D F(0)=I$ (identity matrix), $F(0)=0$. Such mappings are called Keller mappings; it is sufficient to prove $\mathbf{J C}$ only for such mappings.

In this paper, we consider the structure of Keller mappings; this question seems to be among the most important for both proving or rejecting

(C) Petrozavodsk State University, 2019 
the JC. Solving this question and applying the criteria or sufficient conditions of injectivity would, we believe, significantly advance the study of JC.

The complete description of the Keller mappings for $n=2, m=3$ is given in [8].

Theorem A. [8] Let $F$ be a flat polynomial mapping from $P_{3}, F(0)=0$; it is a Keller mappings if and only if $F=A^{-1} \circ g \circ A$, where $g(x, y)=(U(x, y), V(x, y))$,

$$
\begin{aligned}
& U(x, y)=x+\alpha_{2}(x+y)^{2}+\alpha_{3}(x+y)^{3}, \\
& V(x, y)=y-\alpha_{2}(x+y)^{2}-\alpha_{3}(x+y)^{3},
\end{aligned}
$$

$\alpha_{2}$ and $\alpha_{3}$ are any fixed constants, $A$ is a linear homogeneous non-degenerate mapping. All such mappings $F$ are injective.

The set of Keller mappings for $m>3$ is significantly more complicated (see [9]). A similar to Theorem A result was obtained in [6] as a sufficient condition for any $n$ and $m$.

Theorem B. [6] The Jacobian conjecture is true for polynomial mappings $F(X)=\left(A \circ f \circ A^{-1}\right)(X)$, where $X=\left(x_{1}, \ldots, x_{n}\right) \in \mathbb{R}^{n}$, where $A$ is a linear homogeneous non-degenerate mapping, $f=\left(u_{1}, \ldots, u_{n}\right)$,

$$
\begin{aligned}
u_{k}(X)=x_{k}+\gamma_{k}[ & \alpha_{2}\left(x_{1}+\ldots+x_{n}\right)^{2}+ \\
& \left.+\alpha_{3}\left(x_{1}+\ldots+x_{n}\right)^{3}+\ldots+\alpha_{m}\left(x_{1}+\ldots+x_{n}\right)^{m}\right],
\end{aligned}
$$

$\alpha_{j}, \gamma_{k} \in \mathbb{R}$ with $\sum_{k=1}^{n} \gamma_{k}=0$ for $k=1, \ldots, n$. Then $F(X)$ is a Keller mapping.

Theorem B can be significantly generalized:

Theorem C. [6] Let $n \geqslant 2$ and mapping $f(X)=\left(U_{1}, \ldots, U_{n}\right): \mathbb{R}^{n} \rightarrow \mathbb{R}^{n}$ be defined as:

$$
U_{k}(X)=x_{k}+p_{k 2}\left(x_{1}+\ldots+x_{n}\right)^{2}+\ldots+p_{k m}\left(x_{1}+\ldots+x_{n}\right)^{m}=x_{k}+v_{k}(z),
$$

where $z=x_{1}+\ldots+x_{n} ; v_{k}(z)=\sum_{j=2}^{m} p_{k j} z^{j}, k=1, \ldots, n$ and $p_{k j}$ are any constant values that follow the condition $\sum_{k=1}^{n} p_{k j}=0$ for all $j=2, \ldots, m$. Then $\mathbf{J C}$ is true for $f$ and $f=f_{1} \circ \cdots \circ f_{N}$, where $f_{l}(l=1, \ldots, N)$ are polynomial mappings of type $f$ from Theorem $B$. 
The authors of [8] and [6] note that these results remain true also in the complex case.

In connection with Theorem $\mathrm{C}$, there appears

Question 1: whether Theorem $\mathrm{C}$ remains true if the sum $z=x_{1}+$ $+\ldots+x_{n}$ is replaced by $Z=b_{1} x_{1}+\ldots+b_{n} x_{n}$ with an arbitrary vector $B=\left(b_{1}, \ldots, b_{n}\right), B \nVdash(1, \ldots, 1)$ in definition (1) of functions $v_{k}(z)$ ? Here it is natural to assume that $B \nVdash(1,1, \ldots, 1)=: E$, in order not to get mappings of type (1), already described by Theorem $C$. So this is assumed in the sequel. Also, for the same reason, we assume that $B \neq 0$.

This problem is solved by choosing for each mapping $f$ from Theorem $\mathrm{C}$ a non-degenerate matrix $A$, such that the new mapping $\Phi(X)=A^{-1} f(A X)$ has the desired properties. It turns out, that such a matrix does not always exist. The following theorem holds:

Theorem 1. Let $k=1, \ldots, n$ and $P^{(k)}=\left(p_{k 2}, \ldots, p_{k m}\right)$ be $(m-1)$-dimensional vectors, not all null, $m \geqslant 2, \sum_{k=1}^{n} P^{(k)}=0$. Let $\mathbb{R}^{n} \ni B=$ $=\left(b_{1}, \ldots, b_{n}\right) \nVdash(1, \ldots, 1), f(X)$ be from Theorem $C$ is defined using condition (1) by choosing vectors $P^{(k)}$.

1) Assume that in the set of vectors $P^{(k)}, k=1, \ldots, n$ at most $n-2$ are linearly independent; then a non-degenerate matrix $A$, such that

$$
\Phi(X)=A^{-1} f(A X)=X+\left(\begin{array}{c}
q_{12} Z^{2}+\ldots+q_{1 m} Z^{m} \\
\ldots \ldots \ldots \ldots \ldots \ldots \\
q_{n 2} Z^{2}+\ldots+q_{n m} Z^{m}
\end{array}\right)
$$

exists; here $\sum_{k=1}^{n} q_{k j}=0$ for any $j=2, \ldots, m$, and $Z=(X, B)$ denotes the Euclidean scalar product of vectors $X$ and $B$.

2) Assume that in the set of vectors $P^{(k)}, k=1, \ldots, n$, at least $(n-1)$ are linearly independent; then no matrix $A$ satisfies equalities (2) and $\sum_{k=1}^{n} q_{k j}=0$ for any $j=2, \ldots, m$.

Note that if the condition $\sum_{k=1}^{n} p_{k j}=0, j=2, \ldots, m$, is not satisfied in Theorem $\mathrm{C}$, the mapping $f$ defined by formula (1) would not generally be a Keller mapping and $\mathbf{J C}$ would not be true. In this connection, there appears the 
Question 2: in Theorem 1, how important is the condition $\sum_{k=1}^{n} q_{k j}=0$ for polynomial mappings

$$
\Phi(X)=X+\sum_{l=2}^{m} Q_{l} Z^{l}, \text { where } Q_{l}=\left(\begin{array}{c}
q_{1 l} \\
\vdots \\
q_{n l}
\end{array}\right), \quad Z=(X, B),
$$

to be Keller mappings?

The answer to this question is the following

Theorem 2. For any vector $B=\left(b_{1}, \ldots, b_{n}\right)$ and any vectors $Q_{j}$, $j=2, \ldots, m$ from the linear vector space $M$ orthogonal to $B$, the polynomial mapping $\Phi(X)$ from $\left(^{*}\right)$ is a Keller mapping, and the $\mathbf{J C}$ is valid for it.

Theorem $\mathrm{C}$ is a partial case of Theorem 2 for $B=(1, \ldots, 1)$. In Theorem 2 the assumption that the vectors $Q_{j}$ belong to the space $M$, $M \perp B$, is essential.

To describe the structure of the Keller mappings, it is important to study their compositions (see, e.g., [9] and [6]). The third part of this paper is devoted to this question.

\section{Proofs of Theorems 1 and 2.}

Proof of Theorem 1. Let $f$ be the mapping from Theorem $\mathrm{C}$ and a non-degenerate matrix

$$
A:=\left(\begin{array}{cccc}
a_{11} & a_{12} & \ldots & a_{1 n} \\
\vdots & \vdots & \ddots & \vdots \\
a_{n 1} & a_{n 2} & \ldots & a_{n n}
\end{array}\right), \quad A^{-1}:=\left(\begin{array}{cccc}
c_{11} & c_{12} & \ldots & c_{1 n} \\
\vdots & \vdots & \ddots & \vdots \\
c_{n 1} & c_{n 2} & \ldots & c_{n n}
\end{array}\right)=C .
$$

Denote

$$
\begin{gathered}
Y=\left(\begin{array}{c}
y_{1} \\
\vdots \\
y_{n}
\end{array}\right):=A X=\left(\begin{array}{c}
a_{11} x_{1}+a_{12} x_{2}+\ldots+a_{1 n} x_{n} \\
\vdots \\
a_{n 1} x_{1}+a_{n 2} x_{2}+\ldots+a_{n n} x_{n}
\end{array}\right), \\
Z_{*}=y_{1}+\cdots+y_{n}, \quad F(X)=f(A X)=f\left(y_{1}, \ldots, y_{n}\right)=\left(U_{1}(Y), \ldots, U_{n}(Y)\right) .
\end{gathered}
$$

From (1) we get 


$$
\begin{aligned}
& U_{k}(Y)=y_{k}+v_{k}\left(Z_{*}\right)= \\
= & y_{k}+\sum_{j=2}^{m} p_{k j}\left[x_{1}\left(a_{11}+\ldots+a_{n 1}\right)+\ldots+x_{n}\left(a_{1 n}+\ldots+a_{n n}\right)\right]^{j}, k=1, \ldots, n,
\end{aligned}
$$

even more, $\sum_{k=1}^{n} p_{k j}=0$ for all $j=2,3, \ldots, m$. From Theorem $\mathrm{C}$ it follows, that $f(X)$ is a Keller mapping. Therefore, the Jacobian of $\Phi(X)=A^{-1} f(A X)$ is $J_{\Phi}(X) \equiv 1$ and $D \Phi(0)=I$, which means that $\Phi$ is a Keller mapping.

Now denote

$$
a_{11}+\ldots+a_{n 1}=b_{1}^{*}, \ldots, a_{1 n}+\ldots+a_{n n}=b_{n}^{*}, \quad B^{*}=\left(\begin{array}{c}
b_{1}^{*} \\
\vdots \\
b_{n}^{*}
\end{array}\right)
$$

$B^{*} \neq 0$, because $\operatorname{det} A \neq 0$. Let us prove the following statement: A mapping $\Phi$ has the form (2), if and only if a constant $c \neq 0$ exists, such that $B^{*}=B c$.

Indeed, the sufficiency is obvious. Let us check necessity. If there is no such constant $c \neq 0$ that $B^{*}=B c$, equations $z=(X, B)=0$ and $Z_{*}=\left(X, B^{*}\right)=0$ define different hyperplanes $\Pi_{1}$ and $\Pi_{2}$, respectively. Choose a point in $X \in \Pi_{1} \backslash \Pi_{2}$. For such $X$, when equality (2) is valid, we have $\Phi(X)=X$. Choose a $\lambda \rightarrow+\infty$. Then $\Phi(\lambda X)=\lambda X$. Not all $p_{k j}$ are zero, due to assumptions of Theorem 1, so $\|f(A X \lambda)\|$ grows as $\lambda^{r}$, where $r>1$, as $\lambda \rightarrow+\infty$. Therefore, $\|\Phi(\lambda X)\|$ has the same growth order $\lambda^{r}$. The obtained contradiction shows that there exists an $X$ that violates equality (2). Thus $B^{*}=B c$ for some constant $c \neq 0$. This means that $B c=A^{T} E$; here $E$ is the column vector $(1,1, \ldots, 1)$. Let us write the last equality in the form

$$
\left(A^{T}\right)^{-1} B c=C^{T} B c=E .
$$

When condition (3) holds, $Z_{*}$ has the form $Z_{*}=c(X B)=c Z$, so

$$
\begin{gathered}
\Phi(X)=A^{-1} f(A X)=X+C\left(\begin{array}{c}
v_{1}\left(Z_{*}\right) \\
\vdots \\
v_{n}\left(Z_{*}\right)
\end{array}\right)= \\
=X+\left(\begin{array}{c}
v_{1}\left(Z_{*}\right) c_{11}+\ldots+v_{n}\left(Z_{*}\right) c_{1 n} \\
\vdots \\
v_{1}\left(Z_{*}\right) c_{n 1}+\ldots+v_{n}\left(Z_{*}\right) c_{n n}
\end{array}\right)=
\end{gathered}
$$




$$
\begin{aligned}
=X+\left(\begin{array}{c}
Z_{*}^{2} \sum_{k=1}^{n} p_{k 2} c_{1 k}+\ldots+Z_{*}^{m} \sum_{k=1}^{n} p_{k m} c_{1 k} \\
\vdots \\
Z_{*}^{2} \sum_{k=1}^{n} p_{k 2} c_{n k}+\ldots+Z_{*}^{m} \sum_{k=1}^{n} p_{k m} c_{n k}
\end{array}\right)= \\
=X+\left(\begin{array}{c}
q_{12}^{*} Z_{*}^{2}+\ldots+q_{1 m}^{*} Z_{*}^{m} \\
\vdots \\
q_{n 2}^{*} Z_{*}^{2}+\ldots+q_{n m}^{*} Z_{*}^{m}
\end{array}\right)= \\
=X+\left(\begin{array}{c}
q_{1,2} c^{2} Z^{2}+\ldots+q_{1, m} c^{m} Z^{m} \\
\vdots \\
q_{n, 2} c^{2} Z^{2}+\ldots+q_{n, m} c^{m} Z^{m}
\end{array}\right) \\
q_{k, j}^{*}=p_{1 j} c_{k 1}+p_{2 j} c_{k 2}+\ldots+p_{n j} c_{k n}=c^{j} q_{k j},
\end{aligned}
$$

where $k=1, \ldots, n, j=2, \ldots, m$.

In accordance with the problem solved by Theorem 1 , the matrix $C$ we are searching must obey, besides condition (3), also the condition

$$
\sum_{k=1}^{n} q_{k, j}=0 \text {, i. e. } \sum_{k=1}^{n} q_{k, j}^{*}=0
$$

for any $j=2, \ldots, m$. Therefore,

$$
\left\{\begin{array}{c}
p_{12}\left(c_{11}+\ldots+c_{n 1}\right)+\ldots+p_{n 2}\left(c_{1 n}+\ldots+c_{n n}\right)=0 \\
\ldots \ldots \ldots \ldots \ldots \ldots \ldots \ldots \ldots \ldots \ldots \ldots \ldots \ldots \\
p_{1 m}\left(c_{11}+\ldots+c_{n 1}\right)+\ldots+p_{n m}\left(c_{1 n}+\ldots+c_{n n}\right)=0
\end{array}\right.
$$

Denote $C_{+}^{(k)}=c_{1 k}+c_{2 k}+\cdots+c_{n k}, k=1, \ldots, n$, and rewrite (5) as

$$
P^{(1)} C_{+}^{(1)}+P^{(2)} C_{+}^{(2)}+\ldots+P^{(n)} C_{+}^{(n)}=0 .
$$

By definition of the mapping $f$,

$$
P^{(1)}+P^{(2)}+\ldots+P^{(n)}=0 .
$$

Taking this equality into account, rewrite (6) in the form

$$
P^{(2)}\left(C_{+}^{(2)}-C_{+}^{(1)}\right)+\ldots+P^{(n)}\left(C_{+}^{(n)}-C_{+}^{(1)}\right)=0 .
$$


Let us consider two possibilities.

1. There are at least $(n-1)$ linearly independent vectors among $P^{(1)}, \ldots, P^{(n)}$. Without loss of generality we may assume that the vectors $P^{(2)}, \ldots, P^{(n)}$ are independent (otherwise just change the numeration). Then (8) implies

$$
C_{+}^{(l)}-C_{+}^{(1)}=0 \text { for any } l=2, \ldots, n .
$$

Denote the columns of the matrix $C$ by

$$
\widehat{C}_{1}=\left(\begin{array}{c}
c_{11} \\
\vdots \\
c_{n 1}
\end{array}\right), \ldots, \widehat{C}_{n}=\left(\begin{array}{c}
c_{1 n} \\
\vdots \\
c_{n n}
\end{array}\right) .
$$

Equalities (9) mean that vectors $\left(\widehat{C}_{l}-\widehat{C}_{1}\right)$ for $l=2, \ldots, n$ are orthogonal to the vector $E$. On the other hand, from (3) it follows that $\left(\widehat{C}_{l}, B\right)=1 / c$ for $l=1,2, \ldots, n$. Thus $\left(\widehat{C}_{l}-\widehat{C}_{1}\right) \perp B$ for $l=2, \ldots, n$. As $\operatorname{det} C \neq 0$, the vectors $\left\{\left(\widehat{C}_{l}-\widehat{C}_{1}\right)\right\}_{l=2}^{n}$ are linearly independent and a $(n-1)$-dimensional linear space $M_{n-1}$ is spanned on them.

All this implies $B \perp M_{n-1}$ and $E \perp M_{n-1}$. Therefore, $B \| E$, which contradicts the assumption about $B$.

So, in the considered case there is no matrix $A$ that obey conditions (2) and (4): this proves the second part of the Theorem.

2. One case remains to be considered: when any $(n-1)$ vectors from $P^{(k)}, k=1, \ldots, n$, are linearly dependent. Without loss of generality, we can assume that these are $P^{(2)}, \ldots, P^{(n)}$. Then there are numbers $\left\{\lambda_{l}\right\}_{l=2}^{n}$, at least some of which are non-zero, such that $\sum_{l=2}^{n} \lambda_{l} P^{(l)}=0$. For the sake of definiteness we can assume that $\lambda_{2}=\ldots=\lambda_{s}=0$, but $\lambda_{s+1}, \ldots, \lambda_{n} \neq 0$, $s \leqslant n-1$, because the number of linearly independent vectors in $\left\{P^{(k)}\right\}_{k=2}^{n}$ is less than or equal to $n-2$. Denote the $(n-1)$-dimensional linear subspace $\mathbb{R}^{n}$ orthogonal to the vector $E$ by $K_{n-1}$. We noted in the part 1 that the necessary and sufficient condition for the first part of Theorem 1 is the validity of conditions (3), $\left(3^{\prime}\right)$, and (4). Denote $N_{n-2}=K_{n-1} \cap M_{n-1}$; the dimension of $N_{n-2}$ is $(n-2)$, because $E \nVdash B$. Choose in $N_{n-2}$ any system of linearly independent vectors $D_{2}, \ldots, D_{s}$. Then $\left(D_{j}, E\right)=\lambda_{j}=0$ and $\left(D_{j}, B\right)=0$ for $j=2, \ldots, s$. Denote the $(s-1)$-dimensional linear space spanned on the vectors $D_{2}, \ldots, D_{s}$ by $L\left(D_{2}, \ldots, D_{s}\right)$. We can choose the vectors $D_{s+1}, \ldots, D_{s}$ from $M_{n-1} \backslash L\left(D_{2}, \ldots, D_{s}\right)$ so that they, joined 
with the vectors $D_{2}, \ldots, D_{s}$, form the base in $M_{n-1}$; even more, $\left(D_{l}, E\right) \neq$ 0 for $l=s+1, \ldots, n$ (maybe, coordinates of these vectors should be slightly changed for this). Then $D_{l} \perp B$ for any $l=2, \ldots, n$ and $\left(D_{j}, E\right)=0=\lambda_{j}$ for $j=2, \ldots, s$. Therefore, for any $l=s+1, \ldots, n$ there is a constant $\mu_{l} \neq 0$ such that $\left(\mu_{l} D_{l}, E\right)=\lambda_{l}$. Denote $\widehat{D}_{l}:=\mu_{l} D_{l}$ for $l=s+1, \ldots, n$ and $\widehat{D}_{l}:=D_{l}$ for $l=2, \ldots, s$. The vectors $\widehat{D}_{2}, \ldots, \widehat{D}_{s}, \widehat{D}_{s+1}, \ldots, \widehat{D}_{n}$ still form a base of the space $M_{n-1}$. Even more, $\left(\widehat{D}_{l}, E\right)=\lambda_{l}$ for $l=2, \ldots, n$.

Now let us construct the matrix $C$. First, choose the vector $\widehat{C}_{1}=\frac{B}{\|B\|^{2}}$; then $\left(\widehat{C}_{1}, B\right)=1$. Next, choose the vectors $\widehat{C}_{l}, l=2, \ldots, n$, as follows: $\widehat{C}_{l}=\widehat{D}_{l}+\widehat{C}_{1}$. The vectors $\widehat{C}_{1}, \widehat{D}_{2}, \ldots, \widehat{D}_{n}$ form a base in $\mathbb{R}^{n}$; so, $\widehat{C}_{1}, \widehat{C}_{2}, \ldots, \widehat{C}_{n}$ form a base in $\mathbb{R}^{n}$, i. e., $\operatorname{det} C \neq 0$. For the constructed matrix $C$ we have the following: $\left(\widehat{C}_{1}, B\right)=1$ for $l=2, \ldots, n$, $\left(\widehat{C}_{l}, B\right)=\left(\widehat{D}_{l}+\widehat{C}_{1}, B\right)=\left(\widehat{C}_{1}, B\right)=1$, because $\widehat{D}_{l} \in M_{n-1}$, i. e., condition (3) holds. Besides, condition (8) with this matrix $C$ becomes

$$
\begin{gathered}
P^{(2)}\left(\widehat{C}_{2}-\widehat{C}_{1}, E\right)+\ldots+P^{(n)}\left(\widehat{C}_{n}-\widehat{C}_{1}, E\right)=0 \Longleftrightarrow \\
P^{(2)}\left(\widehat{D}_{2}, E\right)+\ldots+P^{(n)}\left(\widehat{D}_{n}, E\right)=0 \Longleftrightarrow \sum_{l=2}^{n} \lambda_{l} P^{(l)}=0 .
\end{gathered}
$$

Therefore, condition (8) holds, and so does condition (4).

Theorem 1 is proved.

Remark 1. Note that in the proof of Theorem 1, the constant $c$ that connects $B$ and the matrix $A$ we were looking for can be made equal to 1 . This can always be done by replacing $A$ by the matrix $A_{1}=\frac{1}{c} A$.

Proof of Theorem 2. To proof Theorem 2, it is enough to establish the existence of a non-degenerate matrix $A$ and a mapping $f$ from Theorem $\mathrm{C}$, such that the equality $\Phi(X)=A^{-1} f(A X)$ holds for any $\left\{q_{k l}\right\}$ that obey the assumptions of Theorem 2. The proof of Theorem 1 shows that this equality holds if and only if the following two conditions hold (notation of Theorem 1 is used):

a) condition (3), and

b) a connection between $\left\{p_{k l}\right\}$ to define the mapping $f$ and $\left\{q_{k l}^{*}\right\}$ from formula $\left(3^{\prime}\right)$.

According to Remark 1, the constant $c=1$ without loss of generality. In the sequel, this is assumed. In a) and b) condition (4) from Theorem 1 is no longer considered. 
Rewrite $\left(3^{\prime}\right)$ in the form

$$
\left(\begin{array}{ccc}
c_{11} & \ldots & c_{1 n} \\
\vdots & \vdots & \vdots \\
c_{k 1} & \ldots & c_{k n} \\
\vdots & \vdots & \vdots \\
c_{n 1} & \ldots & c_{n n}
\end{array}\right)\left(\begin{array}{ccc}
p_{12} & \ldots & p_{1 m} \\
\vdots & \vdots & \vdots \\
p_{k 2} & \ldots & p_{k m} \\
\vdots & \vdots & \vdots \\
p_{n 2} & \ldots & p_{n m}
\end{array}\right)=\left(\begin{array}{ccc}
q_{12} & \ldots & q_{1 m} \\
\vdots & \vdots & \vdots \\
q_{k 2} & \ldots & q_{k m} \\
\vdots & \vdots & \vdots \\
q_{n 2} & \ldots & q_{n m}
\end{array}\right)
$$

Condition (3) becomes

$$
\left(\widehat{C}_{k}-\widehat{C}_{1}\right) \perp B, k=2, \ldots, n,\left(\widehat{C}_{1}, B\right)=1 .
$$

The construction of the matrix $C=A^{-1}$ we are looking for is borrowed from the proof of part $\mathbf{2}$ of Theorem 1: an arbitrary base of $M_{n-1}$ is taken as the vectors $\left(\widehat{C}_{l}-\widehat{C}_{1}\right), l=2, \ldots, n$, and $\widehat{C}_{1}$ is any vector that $\left(\widehat{C}_{1}, B\right)=1$. Then condition a) holds. To fulfil b), choose a suitable mapping $f$ from Theorem C. Taking into account equality (7) from the definition of the mapping $f$, rewrite (10) as follows:

$$
\begin{gathered}
\left\{\begin{array}{c}
p_{2 l}\left(c_{12}-c_{11}\right)+\ldots+p_{n l}\left(c_{1 n}-c_{11}\right)=q_{1 l} \\
\ldots \ldots \ldots \ldots \ldots \ldots \ldots \ldots \ldots \ldots \ldots \ldots \\
p_{2 l}\left(c_{n 2}-c_{n 1}\right)+\ldots \ldots+p_{n l}\left(c_{n n}-c_{n 1}\right)=q_{n l}
\end{array}\right. \\
\Longleftrightarrow\left(\widehat{C}_{2}-\widehat{C}_{1}\right) p_{2 l}+\ldots+\left(\widehat{C}_{n}-\widehat{C}_{1}\right) p_{n l}=Q_{l},
\end{gathered}
$$

where $l=2, \ldots, m$.

Note that $Q_{l} \in M_{n-1}$; so, the numbers $\left\{p_{k l}\right\}_{k=2}^{n}$ are uniquely defined by (11) for any $l=2, \ldots, m$ as coordinates of the vector $Q_{l}$ in the base $\left\{\widehat{C}_{k}-\widehat{C}_{1}\right\}_{k=2}^{n}$. The values $p_{1 l}, l=2, \ldots, m$, are defined from (7). This completely defines the mapping $f$ from Theorem $\mathrm{C}$.

Theorem 2 is proved

Remark 2. Note that, according to the proof, the matrix $A=C^{-1}$ and the mapping $f$ are defined non-uniquely, given a vector $B$ and vectors $Q_{l} \in M_{n-1}, l=2, \ldots, m$.

Remark 3. From Theorem $A$ we see that the assumption that the vectors $Q_{l}$ belong to the orthogonal subspace $M_{n-1}$ in Theorem 2 is important. It can not be weakened, at least in the case $n=2, m=3$.

Also note that Theorem $C$ follows from Theorem 2 for $B=(1, \ldots, 1)$. 
3. An idea of mapping composition. The following theorem shows that the trivial idea of using compositions of Keller mappings for building new classes of such mappings can be quite important.

Theorem 3. Assume that for each natural $s=1, \ldots, r, r \in \mathbb{N}, r \leqslant n$, the polynomial mappings

$$
\begin{gathered}
\Phi_{s}(X)=A_{s}^{-1} f_{s}\left(A_{s} X\right)=X+\left(\begin{array}{c}
q_{12}^{(s)} Z_{s}^{2}(X)+\ldots+q_{1 m}^{(s)} Z_{s}^{m}(X) \\
\vdots \\
q_{n 2}^{(s)} Z_{s}^{2}(X)+\ldots+q_{n m}^{(s)} Z_{s}^{m}(X)
\end{array}\right)=: \\
=: X+\left(\begin{array}{c}
V_{1}^{(s)}(X) \\
\vdots \\
V_{n}^{(s)}(X)
\end{array}\right)=: X+V^{(s)}(X)
\end{gathered}
$$

obey the assumptions of Theorem $2, Z_{s}(X)=\left(X, B_{s}\right)$, the vectors $B_{s}=\left(b_{1}^{(s)}, \ldots, b_{n}^{(s)}\right) \neq 0$. Denote the linear $(n-1)$-dimensional subspace orthogonal to the vector $B_{s}$ by $M_{n-1}^{(s)}$,

$$
Q_{l}^{(s)}=\left(\begin{array}{c}
q_{1 l}^{(s)} \\
\vdots \\
q_{n l}^{(s)}
\end{array}\right), l=2, \ldots, m .
$$

Assume that for any $l=2, \ldots, m$ the following inclusions hold:

$$
Q_{l}^{(1)} \in \bigcap_{s=1}^{r} M_{n-1}^{(s)}, Q_{l}^{(2)} \in \bigcap_{s=2}^{r} M_{n-1}^{(s)}, \ldots, Q_{l}^{(r)} \in M_{n-1}^{(r)} .
$$

Then the polynomial mapping $F(X)=\Phi_{r} \circ \Phi_{r-1} \circ \cdots \circ \Phi_{2} \circ \Phi_{1}$ has the form $F(X)=X+\sum_{s=1}^{r} V^{(s)}(X)$, it is a Keller mapping, and the $\mathbf{J C}$ is true for it.

Proof. Consider

$$
\begin{aligned}
\Phi_{2} \circ \Phi_{1}(X)= & \Phi_{2}\left(X+V^{(1)}(X)\right)=X+V^{(1)}(X)+ \\
+ & \left(\begin{array}{c}
q_{12}^{(2)} Z_{2}^{2}\left(X+V^{(1)}(X)\right)+\ldots+q_{1 m}^{(2)} Z_{2}^{m}\left(X+V^{(1)}(X)\right) \\
\vdots \\
q_{n 2}^{(2)} Z_{2}^{2}\left(X+V^{(1)}(X)\right)+\ldots+q_{n m}^{(2)} Z_{2}^{m}\left(X+V^{(1)}(X)\right)
\end{array}\right),
\end{aligned}
$$


where

$$
\begin{gathered}
Z_{2}\left(X+V^{(1)}(X)\right)=\left(X, B_{2}\right)+\left(V^{(1)}(X), B_{2}\right)= \\
=\left(X, B_{2}\right)+Z_{1}^{2}(X)\left(Q_{2}^{(1)}, B_{2}\right)+\ldots+Z_{1}^{m}(X)\left(Q_{m}^{(1)}, B_{2}\right)=\left(X, B_{2}\right)=Z_{2}(X),
\end{gathered}
$$

because for any $l$ the vectors $Q_{l}^{(1)} \in M_{n-1}^{(2)}$ by the assumptions of the Theorem. Therefore, $\Phi_{2} \circ \Phi_{1}(X)=X+V^{(1)}(X)+V^{(2)}(X)$.

Now consider

$$
\begin{aligned}
& \Phi_{3} \circ \Phi_{2} \circ \Phi_{1}(X)=\Phi_{3}\left[X+V^{(1)}(X)+V^{(2)}(X)\right]= \\
& =X+V^{(1)}(X)+V^{(2)}(X)+\left(\begin{array}{c}
\sum_{l=2}^{m} q_{1 l}^{(3)} Z_{3}^{l}\left[X+V^{(1)}(X)+V^{(2)}(X)\right] \\
\vdots \\
\sum_{l=2}^{m} q_{n l}^{(3)} Z_{3}^{l}\left[X+V^{(1)}(X)+V^{(2)}(X)\right]
\end{array}\right),
\end{aligned}
$$

where

$$
\begin{aligned}
& Z_{3}[X+\left.V^{(1)}(X)+V^{(2)}(X)\right]=\left(X, B_{3}\right)+\left(V^{(1)}(X), B_{3}\right)+\left(V^{(2)}(X), B_{3}\right)= \\
&=\left(X, B_{3}\right)+\left[Z_{1}^{2}(X)\left(Q_{2}^{(1)}, B_{3}\right)+\cdots+Z_{1}^{m}(X)\left(Q_{m}^{(1)}, B_{3}\right)\right]+ \\
&+\left[Z_{2}^{2}(X)\left(Q_{2}^{(2)}, B_{3}\right)+\ldots+Z_{2}^{m}(X)\left(Q_{m}^{(2)}, B_{3}\right)\right]=\left(X, B_{3}\right)=Z_{3}(X)
\end{aligned}
$$

because $Q_{l}^{(1)} \in M_{n-1}^{(3)} \ni Q_{l}^{(2)}$ for any $l=2, \ldots, m$. Therefore,

$$
\Phi_{3} \circ \Phi_{2} \circ \Phi_{1}(X)=X+V^{(1)}(X)+V^{(2)}(X)+V^{(3)}(X) .
$$

Continuing in the same way (if $r>3$ ), we come to the statement of the Theorem 3 .

Theorem 3 significantly widens, compared to Theorem $\mathrm{C}$, the set of described Keller mappings for which $\mathbf{J C}$ is true. Even more, it also seems interesting in the context of the well-known result of Drużkowski (see, e. g., [1]):

Theorem D. [1] In order to verify JC for every $\mathbb{N} \ni n \geqslant 2$, it is sufficient to check it only for polynomial mappings $F$ of the cubic linear form, i. e.,

$$
F(X)=X+\left(\begin{array}{c}
\left(B_{1}, X\right)^{3} \\
\vdots \\
\left(B_{n}, X\right)^{3}
\end{array}\right)
$$


the matrix

$$
\mathcal{B}=\left(\begin{array}{ccc}
b_{1}^{(1)} & \ldots & b_{n}^{(1)} \\
\vdots & \ddots & \vdots \\
b_{1}^{(n)} & \ldots & b_{n}^{(n)}
\end{array}\right)
$$

built of the vectors $B_{s}=\left(b_{1}^{(s)}, \ldots, b_{n}^{(s)}\right), s-1, \ldots, n$, may be considered nilpotent: $\mathcal{B}^{2}=0$.

Let us write down Theorem 3 for the case $r=n, m=3, Q_{2}^{(s)}=0$ for $s=1, \ldots, n$ :

Corollary 1. Using the notation of Theorem 3, let $B_{s}, s=1, \ldots, n$, the system of linearly dependent vectors

$$
Q_{3}^{(1)} \in \bigcap_{s=1}^{n} M_{n-1}^{(s)}, Q_{3}^{(2)} \in \bigcap_{s=2}^{n} M_{n-1}^{(s)}, \ldots, Q_{3}^{(n)} \in M_{n-1}^{(n)} .
$$

Then the polynomial mapping

$$
F(X)=X+Q_{3}^{(1)} Z_{1}^{3}(X)+Q_{3}^{(2)} Z_{2}^{3}(X)+\ldots+Q_{3}^{(n)} Z_{n}^{3}(X)
$$

is a Keller mapping and $\mathbf{J C}$ is true for it.

Note that $\operatorname{dim}\left(\bigcap_{s=1}^{n} M_{n-1}^{(s)}\right) \geqslant 1$, because the vectors $B_{s}$ in the Corollary 1 are linearly dependent. Corollary 1 allows generating sets of Keller mappings of the form (12). The following example is inspired by this Corollary and Theorem D.

Example. Let $\mathcal{B}$ be a nilpotent matrix, $\mathcal{B}^{2}=0$;

$$
\mathcal{B}=\left(\begin{array}{ccccc}
b_{1}^{(1)} & \ldots & b_{k}^{(1)} & \ldots & b_{n}^{(1)} \\
\vdots & \ddots & \vdots & \ddots & \vdots \\
b_{1}^{(n)} & \ldots & b_{k}^{(n)} & \ldots & b_{n}^{(n)}
\end{array}\right)
$$

i. e., for any $k=1, \ldots n$

$$
\left\{\begin{array}{l}
b_{1}^{(1)} b_{k}^{(1)}+b_{2}^{(1)} b_{k}^{(2)}+\ldots+b_{n}^{(1)} b_{k}^{(n)}=0 \\
\ldots \ldots \ldots \ldots \ldots \ldots \ldots \ldots \ldots \\
b_{1}^{(n)} b_{k}^{(1)}+b_{2}^{(n)} b_{k}^{(2)}+\ldots+b_{n}^{(n)} b_{k}^{(n)}=0
\end{array}\right.
$$


In the Corollary 1 , choose the vectors $\left(b_{1}^{(s)}, \ldots, b_{n}^{(s)}\right), s=1, \ldots, n$, for the vectors $B_{s}$. Let

$$
Q_{3}^{(1)}=\left(b_{1}^{(1)}, \ldots, b_{1}^{(n)}\right), Q_{3}^{(2)}=\left(b_{2}^{(1)}, \ldots, b_{2}^{(n)}\right), \ldots, Q_{3}^{(n)}=\left(b_{n}^{(1)}, \ldots, b_{n}^{(n)}\right) .
$$

From (13) it follows that $Q_{3}^{(k)} \perp B_{s}$ for any $k, s=1, \ldots, n$. Therefore, all assumptions in Corollary 1 are fulfilled. So,

$$
F(X)=X+\left(\begin{array}{c}
b_{1}^{(1)} \\
\vdots \\
b_{1}^{(n)}
\end{array}\right)\left(B_{1}, X\right)^{3}+\ldots+\left(\begin{array}{c}
b_{n}^{(1)} \\
\vdots \\
b_{n}^{(n)}
\end{array}\right)\left(B_{n}, X\right)^{3}
$$

are injective Keller mappings for any nilpotent matrix $\mathcal{B}$.

Acknowledgment. The author is grateful to the referees for their useful comments and suggestions.

The work is supported by the Russian Science Foundation under grant 17-11-01229.

\section{References}

[1] Drużkowski L. M. On the global asymptotic stability problem and the Jacobian conjecture. Control and Cybernetics, 2005, vol. 34, no. 3, pp. 747-762.

[2] van den Essen A. Polynomial automorphisms and the Jacobian conjecture. vol. 190 of Progress in Mathematics, Birkhäuser Verlag, Basel, 2000.

DOI: $10.1007 / 978-3-0348-8440-2$

[3] Keller O. H. Ganze Cremona-Transformationen. Monatshefte Math. Phys., 1939, vol. 47. pp. $299-306$.

DOI: https://doi.org/10.1007/BF01695502

[4] Kulikov V. S. Generalized and local Jacobian problems. (Russian) Izv. Ross. Akad. Nauk Ser. Mat., 1992, vol. 56, no. 5, pp. 1086-1103; translation in Russian Academy of Sciences. Izvestiya Mathematics, 1993, vol. 41, no. 2, pp. $351-365$.

[5] Moh T. T. On the global Jacobian conjecture and the configuration of roots. J. reine und angew. Math., 1983, vol. 340, pp. 140-212.

[6] Ponnusamy S., Starkov V. V. The Jacobian Conjecture and Injectivity Conditions. Bulletin of the Malaysian Mathematical Sciences Society, 2018, vol. 41, no. 4, pp. $2099-2115$.

DOI: https://doi.org/10.1007/s40840-018-0626-9 
[7] Smale S. Mathematical Problems for the Next Century. Math. Intelligencer., 1998, vol. 20, no. 2, pp. $7-15$.

DOI: https://doi.org/10.1007/BF03025291

[8] Starkov V. V. Jacobian conjecture, two-dimensional case. Probl. Anal. Issues Anal., 2016, vol. 5 (23), no. 2, pp. 69-78.

DOI: $10.15393 / j 3$.art. 2016.3510

[9] Starkov V. V. Structure of Keller mappings,two-dimensional case. Probl. Anal. Issues Anal., 2017, vol. 6 (24), no. 1, pp. 68-81.

DOI: $10.15393 / j 3$.art.2017.3870

[10] Wang S. S.-S. A Jacobian criterion for separability. J. of Algebra, 1980, vol. 65 , no. 2 , pp. $453-494$.

DOI: https://doi.org/10.1016/0021-8693(80)90233-1

Received August 23, 2019.

In revised form, October 25, 2019.

Accepted October 25, 2019.

Published online November 1, 2019.

Petrozavodsk State University

33 Lenina pr., Petrozavodsk 185910, Russia

E-mail: VstarV@list.ru 